\title{
Timo Tohidipur
}

\section{Iran und die Narrative west-östlicher Begegnung}

Der Iran definiert sich zunächst über seine lange Geschichte als antikes Weltreich und später, noch bis an die Schwelle zum 19. Jahrhundert, als regionale Großmacht. ${ }^{1}$ Umso wirkmächtiger sind die Schatten, die die nachfolgenden, weniger ruhmreichen Begegnungen mit dem europäisch-atlantischen Staatengefüge im iranischen Streben nach Unabhängigkeit und Sicherheit hinterlassen haben und die bis in die Gegenwart überaus präsent sind. Dabei war der Iran nie Kolonie und spielt folglich in der Literatur zur europäisch-atlantischen Kolonialgeschichte ebenso wenig eine Rolle wie in der postkolonialen Debatte. Gleichwohl haben sich diverse externe Einflussnahmen im Kontext west-östlicher Begegnung in das kollektive Gedächtnis des Iran eingeschrieben, die auch für das zeitgenössische politische Miteinander relevant bleiben. Im Fokus steht hier nicht eine weitere Stellungnahme zur aktuellen Konfliktsituation, sondern vielmehr die Aufarbeitung von Lesarten eines quasi-postkolonialen Selbstfindungsprozesses, der zugleich das grundlegende Interesse der iranischen Gesellschaft gegenüber dem Anderen inkludiert.

\section{Annäherung an ein imperiales Szenario}

Es greift zu kurz, will man die vielfältigen internationalen Verstimmungen und Konflikte, die sich fortwährend mit Bezug auf den Iran offenbaren, allein auf die Auswirkungen der islamischen Revolution des Jahres 1978/1979 stützen. Die islamische Revolution ist selbstverständlich der entscheidende Faktor für die aktuelle Verfasstheit des Iran, bildet aber gleichzeitig einen vorläufigen Schlusspunkt in den Bestrebungen nach politischer Emanzipation von innerer und äuBerer Subordination und politischer Fremdbestimmung des Landes.

\section{Das dunkle 19. Jabrbundert}

So war der Iran bereits seit Mitte des 19. Jahrhunderts in den Kampf um die kolonialen Bestrebungen Europas im sogenannten „Nahen Osten“ verstrickt. ${ }^{2}$ Schon die - mittlerweile etablierte - Vokabel „Naher Osten“ impliziert dabei prägnant die eurozentrische Sichtweise auf den asiatischen Kontinent, der an Nähe und Ferne zu Europa gemessen und nach Interessensgebieten vermessen wird. ${ }^{3}$ Seine zentrale Lage in Westasien und sein Rohstoffreichtum ließen den Iran zum begehrten Ziel werden. Am Anfang stand dabei die Begegnung mit einer

1 Schatt, Persien und Mittelasien bis zum Beginn des 19. Jahrhunderts, in: Pleticha (Hrsg.), Weltgeschichte. Alte Völker neue Staaten, S. 54 ff.; Wiesehöfer, Das antike Persien, 2005; Axworthy, Iran. Weltreich des Geistes, 2011.

2 Mehdi Tohidipur, Das Werden und der Inhalt der iranischen Verfassung, VRÜ 1974, S. 189; Gurland, Wirtschaft und Gesellschaft im Übergang zum Zeitalter der Industrie, in: Mann (Hrsg.), Weltgeschichte, Band 8, 1960, S. 280.

3 Vgl. hierzu die ähnlich gelagerte Kritik am Begriff „Mittlerer Osten“ als unzulänglichen Sammelbegriff für „das Andere“ und seine eurozentrische Zuschreibung Al-Nowaihi, The „Middle East“?... Or/Arabic Literature and the Postcolonial Predicament, in: Schwarz/Ray (Hrsg.), A Companion to Postcolonial Studies, 2005, S. $282 \mathrm{f}$. 
europäisch-asiatischen Macht, mit Russland, das nach vielen Jahren wechselvoller militärischer und politischer Auseinandersetzungen mit dem $\operatorname{Iran}^{4}$ zuletzt mit dem Frieden von Turkmantschai 1828 vom Iran den Kaukasus, Georgien und Teile Aserbeidschans erlangte, sowie später noch die für die persische Kultur so wichtigen Fürstentümer Buchara und Samarkand. ${ }^{5}$ Zudem gewährten nachfolgend abgeschlossene Handelsabkommen den russischen Kaufleuten ungehinderten Marktzugang, wobei sie nicht der iranischen Rechtsprechung unterworfen waren. Rückschläge waren auch gegenüber Großbritannien zu verzeichnen, das im Wechsel mit dem Gegenspieler Frankreich über eine lange Zeit hinweg zunächst Handelspartner des Iran war, dann aber beispielsweise Mitte des 19. Jahrhunderts erfolgreich militärisch verhinderte, dass Teile Afghanistans vom Iran zurückerobert wurden. Doch Russland und insbesondere Großbritannien etablierten und vertieften neben dem militärischen Druck auch ökonomischtechnologische Abhängigkeiten des nicht industrialisierten $\operatorname{Iran}^{6}$ und deklassierten das Land in diesem Kontext als „Entwicklungsland“. Folgenreich war hierbei, dass der britischen Regierung fortlaufend weitgehende Handelskonzessionen eingeräumt wurden, so letztlich auch das Recht auf Errichtung von Bergwerken, Fabriken und landwirtschaftlichen Betrieben sowie den daraus abzuschöpfenden Gewinn, wodurch der Iran seine durch Ressourcen gestützte unabhängige Wirtschaftskraft fast gänzlich verlor. ${ }^{7}$ Insoweit ist hier eine Parallele zu der gesamten westasiatischen Region zu erblicken, deren Länder zu Rohstofflieferanten degradiert wurden, während Großbritannien und Frankreich zum „vorderorientalischen Bankier“ avancierten. ${ }^{8}$ Dabei verbesserte sich die ökonomisch-technologische Situation im Iran nicht, der Abstand zu den europäischen Staaten vergrößerte sich vielmehr, nicht zuletzt aufgrund der diversen vertraglichen Privilegien, gefördert durch innenpolitische Regierungsschwäche. ${ }^{9}$ Die Regierungsschwäche äußerte sich im Ausverkauf des Landes, sozusagen in der Einladung ausbeuterischer Bestrebungen durch korrupte Herrscher.

\section{Formelles und informelles Empire}

Der Iran wurde, wenngleich nie formell zur Kolonie erklärt, im Strudel der Interessensrivalität zwischen Großbritannien und Russland in eine russische Interessenssphäre im Norden und eine britische im Süden aufgeteilt. ${ }^{10}$ Dabei blieb die formelle Unabhängigkeit stets bestehen. Doch auch ohne unter direkte Fremdherrschaft zu fallen, wurde selbst der Iran nun Teil des „informellen Empire“ Großbritanniens in Westasien, das auf die Sicherung der imperialen Verbindungsrouten von Ostasien bis zum Mittelmeer bedacht war. ${ }^{11}$ Im Gegensatz zur kolonialen Herrschaft, verstehbar als formelles Empire, bei dem der einheimische Machthaber durch einen fremden ersetzt wird, der mit seiner Kolonialverwaltung die zentralen Hoheitsfunktionen im Kolonialstaat übernimmt, bleibt beim informellen Empire der Zielstaat als selbständiges Gemeinwesen mit seinem eigenen politischen System und dem entsprechenden souveränen Handlungsspielraum bestehen. ${ }^{12}$ Gleichwohl werden Privilegien, die vertraglich garantiert

Sehr ausführlich hierzu Kazemzadeh, Russia and Britain in Persia, 1864-1914. A Study in Imperialism, 1968 und Axworthy (Fn. 4), S. 190 f.

5 Schweizer, , Iran. Drehscheibe zwischen Ost und West, 5. Aufl. 2005, S. 237 f.

6 Tohidipur (Fn. 2), S. 189.

7 Schweizer (Fn. 5), S. 241; Axworthy (Fn. 4), S. 201.

8 Watrin, Machtwechsel im Nahen Osten, 1989, S. 63.

9 Axworthy (Fn. 4), S. 199.

10 Tohidipur (Fn. 2), S. 189; Reinhard, Kleine Geschichte des Kolonialismus, 2. Aufl. 2008 , S. 243 f.

11 Watrin (Fn. 8), S. 58 f., 73 f.; Bush, Imperialism and Postcolonialism, 2006, S. 201.

12 Osterhammel, Kolonialismus, 6. Aufl. 2009, S. 25; Bush (Fn. 11), S. 45 f. 
werden und die sich auch auf die Anwendbarkeit des einheimischen Rechts, d.h. den Schutz ausländischer Staatsangehöriger vor der unmittelbaren Geltung einheimischer Gesetze beziehen, verbrieft, ${ }^{13}$ was für britische Staatsangehörige, wie zuvor schon für russische und später für amerikanische Staatsangehörige auch erreicht wurde. ${ }^{14}$ Wie fließend die Grenzen zum Verständnis von Kolonialisierung sein können, zeigt eine überlieferte Äußerung eines führenden britischen Kolonialbeamten, wonach es sich beim Iran um die „vollständigste und außerordentlichste Überlassung der gesamten Wirtschaftsquellen eines Königreiches an Ausländer" gehandelt hätte. ${ }^{15}$ Gemeinsam ist allen Ansätzen das Erlebnis der, teils gewaltsamen, Unterwerfung unter die Diktate des Fremden. ${ }^{16}$ Naheliegend ist daher die Anwendbarkeit des „dynamischen Komplementärbegriffs“ zum eher statisch gedachten Kolonialismus, des Imperialismus, verstehbar als die Anstrengungen, die auf die Herstellung einer informellen oder formellen kolonialen Politik ausgerichtet sind, also der Kontrolle eines fremdes Landes unter wirtschaftlicher, politischer und gegebenenfalls ideologischer Ausnutzung der Entwicklungsdifferenz. ${ }^{17}$ Diese Annäherung an ein imperiales Szenario eröffnet einen anderen Blick auf mehrere Episoden konfliktgeladener Auseinandersetzung mit externen Hegemonieansprüchen und politischen Emanzipationsbestrebungen. Insoweit erscheint es angebracht, den Iran in eine postkoloniale Debatte einzubeziehen, zumal der Begriff des „Postkolonialen“ sich ja gerade nicht nur auf die Regionen beschränkt, die bis zum Zweiten Weltkrieg formelle Kolonien waren. ${ }^{18}$ Die Gründe werden anhand dreier exemplarischer Episoden skizziert.

\section{Episode 1 - Konstitutionsrevolution als Versuch innerer und äußerer Befreiung}

Die als demütigend verstandenen Friedensverträge mit Russland, die zunehmende Präsenz ausländischen - dabei primär europäischen - Einflusses auf Wirtschaft und Politik und die mangelnde Entwicklung der Lebenssituation im Land führte im 19. Jahrhundert zu steigendem Unmut in weiten Teilen der Bürgerschaft.

\section{Aufbegebren gegen Konzessionshandel}

In den Auseinandersetzungen um die Folgen der russisch-iranischen Friedensverträge hatten schon Mitte des 19. Jahrhunderts Teile des Volkes und insbesondere der einflussreichen Bazarkaufleute erfolgreich zu politischen Protesten aufgerufen. ${ }^{19}$ Die kadscharische Führung unter Fateh Ali Schah's Sohn Mohammed Schah und seinem Nachfolger Nasir ad Din vermochte die Interessen des Landes aus der Sicht weiter Teile des Volkes nicht effektiv zu wahren, auch nicht gegen die Einmischung von Großbritannien und Russland in die Regierungsgeschäfte bis hin zur Einflussnahme auf die Herrschernachfolge. ${ }^{20}$ Der jeweilige Schah versuchte vielmehr, von diesen Umständen finanziell zu profitieren. Zugleich mit den Fischereirechten im Kaspischen Meer für die russische Regierung, erlangte

Balfour-Paul, The Ende of Empire in the Middle East, 1991, S. 96; Axworthy (Fn. 4), S. 254.

15 Vgl. Schweizer (Fn. 5), S. 250, der diese Äußerung Lord Curzon zuschreibt, dem ehemaligen Vizekönig von Indien und späteren britischen Außenminister.

16 Kapferer, Islamische Renaissance, Islamische Revolution, Islamische Theokratie, APuZ 1980, S. 4.

17 Reinhard (Fn. 10), S. 1; Bush (Fn. 11), S. 45 f.

18 Zum Begriff und zur Kritik vgl. Robbins, Race, Gender, Class, Postcolonialism, in: Schwarz/Ray (Hrsg.), A Companion to Postcolonial Studies, 2005, S. 556 (564 f.).

19 Axworthy (Fn. 4), S. 195, 197.

20 Katouzian, State and Society in Iran, 2006, S. 26; Axworthy (Fn. 4), S. 207. 
die britische ihrerseits Konzessionen zur Errichtung einer Nationalbank, der

Imperial Bank of Persia, und zum Bau von Fabriken und Bergwerken, sicherte sich zudem die Ausbeutung der iranischen Ölreserven über ihre Konzessionen, die der Anglo-Iranian Oil Company (AIOC) zugeschrieben wurden, ein zu $100 \%$ britisches Unternehmen. ${ }^{21}$ So wird in der Gesamtschau durchaus überzeugend von einer Situation der „Strangulierung“ des Iran gesprochen. ${ }^{22}$ Unternehmen der AIOC, wie etwa die damals größte Ölraffinerie der Welt in Abadan, funktionierten quasi wie ein kolonialer Außenposten - mit eigenen, separierten Stadtteilen für die britischen Arbeitnehmer inklusive Swimmingpools und Tennisplätzen und slumähnlichen Bereichen für die iranischen Arbeitnehmer, und auch die Unternehmen selbst waren quasi-extraterritoriales Gebiet, zu dem der iranische Staat, die Behörden, keinen wirklichen Zugang hatten. ${ }^{23} \mathrm{Zu}$ weit ging dann aber der iranischen Bevölkerung, dass der Schah Großbritannien 1890 eine exklusive Tabakkonzession erteilte, die den konkurrenzlosen Handel mit Tabak erlaubte, mit anteiliger Beteiligung am Gewinn für sich selbst. ${ }^{24}$ Hiergegen formierte sich eine, selbst durch die Rücknahme der Konzession nicht mehr zu stoppende nationale Protestbewegung gegen den Schah, den „Lakai“ der „Ungläubigen“, ${ }^{25}$ der die Benachteiligung des iranischen Handels und der Bürger erlaube. ${ }^{26}$ Unterstützt durch weite Teile der Religionsgelehrten - der Schiismus persischer Prägung und die ihm zugrundeliegenden politisch-ethischen Grundsätze waren stets präsente Maßstäbe für Volksbewegungen - und getragen von den Kaufleuten im Bazar, Intellektuellen und einer neu entstandenen Bürgerschaft, kam das Land zwischen 1905-1906 nicht zur Ruhe, ehe eine beratende Nationalversammlung einberufen und eine Verfassung ausgearbeitet wurde.

\section{Konstitutionelle Bewegung}

Schon bei dieser letztlich ersten „modernen“ Revolution in Asien spielten die schiitischen Rechtsgelehrten also bereits eine bedeutende Rolle. ${ }^{27}$ Die Aufgeschlossenheit gegenüber auswärtigen Einflüssen und vor allem europäischen Gesellschaftsformen und einer als europäisch verstandenen Modernität zeigte sich hierbei in der deutlichen Orientierung der ersten iranischen Verfassung an europäischem Gedankengut ${ }^{28}$ und insbesondere der belgischen Verfassung. ${ }^{29}$ Doch diese konstitutionelle Entwicklung war erst der Beginn einer Auseinandersetzung um die Macht im Staat Iran, die insbesondere zur Verabschiedung des bedeutenden Verfassungszusatzes von 1907 mit Festschreibung des Islam als Staatsreligion führte. Die konstitutionelle Bewegung war eine nationale Bewegung, die den Interessen des Schahs, zu dieser Zeit Muhammad-Ali-Schah, widersprach, der sich durch breite Unterstützung von Russland und Großbritannien auch mit Gewaltaktionen gegen die Bewegung stellte, wodurch diese entscheidend geschwächt wurde. ${ }^{30}$ Diese militärischen Aktionen führten letztlich gar zur Zerstörung des iranischen Parlaments und zur Ermordung von Abge-

Watrin (Fn. 8), S. 65; Halm, Der schiitische Islam. Von der Religion zur Revolution, 1994, S. 139; Schweizer (Fn. 5), S. 250; Axworthy, Iran. Weltreich des Geistes, S. 208.

22 Kazemzadeh (Fn. 4), S. 581 mit einer sehr ausführlichen Darstellung der Gesamtsituation.

23 Kinzer, Im Dienste des Schah. CIA, MI6 und die Wurzeln des Terrors im Nahen Osten, 2009, S. $146 \mathrm{f.}$

24 Schweizer (Fn. 5), S. 230; Axworthy (Fn. 4), S. 210; Kazemzadeh (Fn. 4), S. 248 ff.

25 Hierzu Schweizer (Fn. 5), S. 250; Kazemzadeh (Fn. 4), S. $261 \mathrm{ff}$.

26 Ausführlich Tohidipur (Fn. 2), S. 190 ff.; Halm (Fn. 21), S. 139 f.

27 Arjomand, The Kingdom of Jurists. Constitutionalism and the Legal Order in Iran, in: Grote/Roeder (Hrsg.), Constitutionalism in Islamic Countries, 2012, S. 147; Bayat, Iran's First Revolution, 1991, S. 253.

28 Molavi, The Soul of Iran, 2002, S. 200.

29 Katouzian, Iranian History and Politics. The Dialectic of State and Society, 2003, S. 117.

30 Cambridge History of Iran, Band 7, 2008, S. 343. 
ordneten. ${ }^{31}$ Auch wenn sich der Schah schon wegen dieser Unterdrückung der nationalen iranischen Bewegung nicht lange halten konnte, vermochte sich die konstitutionelle Bewegung mit ihren bürgerlichen Bestrebungen, vor allem gegen die Interessen der imperialistisch agierenden Staaten Russland und Großbritannien, nicht entscheidend politisch zu etablieren, ${ }^{32}$ wodurch sich nach den Wirren des Ersten Weltkrieges mit Reza-Khan-Pahlavi ein iranischer Kaiser (Schah-inSchah) durch das Parlament krönen lassen konnte, der sodann das Parlament auf seine rein formale Präsenz zu reduzieren vermochte ${ }^{33}$ und das Land nach seinen Vorstellungen und den Vorstellungen der britischen Regierung autoritär formte. Die Konstitutionsrevolution zielte also in erster Linie auf Befreiung von korrupter Herrschaft, die ihrerseits durchdrungen war von äußerer, imperialer Einflussnahme und durch deren überbordendes Konzessionsverlangen erst den Auslöser für das Aufbegehren setzte. Der zumindest vorübergehende Sieg der europäischen Staaten mit ihren Interessen bedeutete zugleich eine Niederlage des eigenständigen gesellschaftlichen Fortschritts im Kontext der nach nationaler Selbstbestimmung strebenden Kräfte, ${ }^{34}$ die - und dies zeigt die Ambivalenz der Situation - ihrerseits europäische Gesellschaftsmodelle und Verfasstheiten zum Vorbild hatten.

\section{Episode 2-der Coup d'état 1953}

Die kolonialen oder quasi-kolonialen Strukturen in Asien sind nach dem Zweiten Weltkrieg nicht mehr in dieser Deutlichkeit aufrecht zu erhalten und wurden durch die - größtenteils erfolgreiche - Installierung despotischer Regime abgelöst, die dann in Abhängigkeit von ihren Gönnern, also den europäischen Staaten oder den USA, zu agieren hatten. ${ }^{35}$ Gegen diesen „Kolonialismus mit anderen Mitteln" 36 formierte sich in vielen Staaten eine nationale Bewegung, die auch im Iran wieder aufflammte.

\section{Gestenerte Zerschlagung demokratischer Strukturen}

Einem Ideenpool von Verschwörungstheoretikern scheinen dann die Vorgänge im Jahr 1953 entsprungen zu sein, die das Verhältnis des Iran zu den USA dauerhaft und entscheidend prägen sollten. Doch mittlerweile ist der Sturz der vom Volk gestützten Regierung des Premierministers Mossadegh und die Re-Installierung des Schah Pahlevi, des Sohns von Reza Khan, durch den amerikanischen Geheimdienst CIA unter Mithilfe des britischen MI6 unter dem Decknamen „Operation Ajax“ umfassend aufgearbeitet. ${ }^{37}$ Ironischerweise leitete gerade diese Operation, die als eine der wenigen wirklich „erfolgreichen“ Regime-ChangeOperationen des CIA angesehen wird, ${ }^{38}$ eine destruktive politische Entwicklung im Verhältnis der Regierungen von Iran und den USA ein, die dauerhafter war

32 Katouzian (Fn. 29), S. 117; Tohidipur (Fn. 2), S. 192.

33 Tohidipur (Fn. 2), S. 193 f.; Halliday, Iran. Analyse einer Gesellschaft im Entwicklungskrieg, 1979, S. $29 \mathrm{ff}$.

34 Baraki (Fn. 31), S. 18.

35 Fernández-Armesto, Millennium. Die Weltgeschichte unseres Jahrtausends, 1998, S. 631 ff.

36 Baraki (Fn. 31), S. 9.

37 Kinzer (Fn. 23); Petherick, The CIA in Iran. The 1953 Coup and the origin of the US-Iran Divide, 2006; Bayandor, Iran and the CIA. The Fall of Mosaddeq Revisited, 2010; Weiner, CIA. Die ganze Geschichte, 2009, S. 122-136; Prashad, The Darker Nations. A People's History of the Third World, 2007, S. 75 ff. 
als die kurzfristigen Ziele der Operation selbst. Der Einfluss der britischen Regierung im Iran war durch die Nationalisierungsbewegung geschwächt, ebenso wie in anderen Staaten der Region. ${ }^{39}$ Jede Unterstützung oder auch nur Zulassung der Entwicklung demokratischer Strukturen durch nationale Bewegungen bedeutete stets den weiteren Verlust von Einfluss. ${ }^{40} \mathrm{Im}$ Kontrast zu der Vorstellung, dass die Etablierung freiheitlich-demokratischer Regime im Sinne der internationalen Gemeinschaft sei, wurde in den nationalen Bewegungen umso mehr eine Gefahr gesehen, als mit der Loslösung der ehemaligen Kolonien oder auch kontrollierter Staaten wie Iran von den imperialen Strukturen der europäischen Staaten zugleich die Rohstoffsicherung verloren zu gehen drohte. Der aus einer erstarkten nationalen Bewegung hervorgegangene und in breiten Teilen der Bevölkerung sehr beliebte iranische Premierminister Mossadegh brachte das Parlament 1951 mit Blick auf die nationale Integrität Irans dazu, die iranische Ölindustrie zu verstaatlichen und sich so dem bevormundenden Zugriff von Großbritannien zu entziehen. Die Folgen waren ein Handelsboykott durch Großbritannien und eine Weigerung wirtschaftlicher Unterstützung durch die USA, auf die Mossadegh gehofft hatte. ${ }^{41}$ Noch 1951 wandte sich Mossadegh in einer leidenschaftlichen Rede vor dem UN-Sicherheitsrat vornehmlich an Großbritannien und die USA und zeigte auf, dass Iran zwar wesentlich zur Versorgung der Welt mit Öl beitrage, dies aber keine ausreichenden Vorteile bei den Lebensbedingungen, beim technischen Fortschritt oder der industriellen Entwicklung des Iran gebracht habe, und da die Erdölreserven des Landes, ebenso wie „seine Erde, seine Flüsse und seine Berge Eigentum des iranischen Volkes“ seien, hätten auch allein die Menschen im Iran darüber zu entscheiden. ${ }^{42}$ In fortlaufender Auseinandersetzung mit dem Schah, der auf einen Kompromiss mit der britischen Regierung hoffte, wurde Mossadegh zunächst abgesetzt und durch den kaisertreuen General Zahedi ersetzt, dann durch den revolutionären Druck auf den Straßen der iranischen Städte gegen den Willen des Schah aber wieder eingesetzt. Die hierbei entscheidende Unterstützung durch die Tudeh-Partei, die als Arbeiterpartei zwar kommunistisch war, aber in aktivistischer und intellektueller Hinsicht die nationale Befreiungsbewegung unterstützte, nutzten Großbritannien und die USA, um die gesamte iranische Nationalbewegung als Teil einer internationalen kommunistischen Bedrohung zu konterkarieren ${ }^{43}$ und sich so letztlich auf diesem Wege selbst für die Re-installation der absoluten Monarchie des Schah zu legitimieren. Dabei musste der Schah selbst, so die übereinstimmende Quellenlage, erst noch von Seiten der amerikanischen Regierung zur Beteiligung überzeugt werden, da er sich seines dann folgenden Legitimationsverlustes beim Volk bewusst zu sein schien, was wiederum die Bedeutung des amerikanischen Einflusses bei dieser Operation verdeutlicht. ${ }^{44}$ Die eingesetzten Mittel des amerikanischen Geheimdienstes reichten dabei von „gekauften“ Demonstrationen und Plünderungen, ${ }^{45}$ medialen Hetzkampagnen gegen Mossadegh bis hin zu inszenierten terroristischen Anschlägen - im Namen der Tudeh-Partei - auf iranische Geistliche, die so für den Schah gewonnen werden sollten, was auch ge-

45 Sehr anschaulich berichtet Weiner auf der Basis von CIA-Dokumenten, dass die gekauften Plünderer, die als „kommunistische Schlägerhorde“ auftraten, Brände legten und Regierungsinsignien zertrümmerten, dann von den „echten“ Tudeh-Partei Mitgliedern zum Aufhören bewegt wurden, Weiner (Fn. 37), S. 132 f. 
lang. ${ }^{46}$ Auf dieser Basis putschte dann das iranische Militär gegen Mossadegh erfolgreich und ebnete den Weg zurück zur absoluten Monarchie.

\section{Folgenreiche Reinstallation des Schab}

Der Machtwechsel zurück zum Schah war dabei mehr als nur eine politische Randnote, mehr als das Obsiegen einer zuvor oppositionellen politischen Richtung. Vielmehr wurden viele politische Gegner des erstarkten Kaisers im Nachgang verhaftet, gefoltert und hingerichtet. ${ }^{47}$ Die USA profitierten nun - an der Stelle Großbritanniens - umfangreich von den Erdölförderungen und engagierten sich auch am militärischen Aufbau des Iran, und die CIA half beim effektiven Aufbau des iranischen Geheimdienstes SAVAK, der für das System innerer Sicherheit, auch vor oppositioneller politischer Betätigung, eine bedeutende Rolle spielen sollte. Überaus lukrativ waren die Waffengeschäfte mit dem nach Militarisierung und Aufrüstung strebenden Schah. ${ }^{48}$ In dieser Zeit der deutlichen Einbindung des Iran in die politische Architektur der westlichen Staatengemeinschaft liegen wohl auch die Anfänge des heute umstrittenen Atomprogramms. ${ }^{49}$ Dabei wurden in den 60er und 70er Jahren, neben der wirtschaftlichen Entwicklung, im Iran umfangreiche bildungspolitische und soziale Reformen durchgeführt, die generell durch den erleichterten Zugang zu staatlichen Schulen und Universitäten das Bildungsniveau entscheidend verbesserte und damit auch zur Herausbildung einer gut ausgebildeten Mittelschicht führte. ${ }^{50}$ Während das Staatsprogramm nach 1953 nach außen für die Idee eines „modernen“ Iran zu stehen schien, stand dem die repressive Struktur des Regimes des Schah entgegen, der jenseits eigener Reformideen keine oppositionellen Strukturen gleich welcher Art duldete und nicht zuletzt auch an seiner eigenen finanziellen Absicherung arbeitete. ${ }^{51}$ Die wirtschaftliche und soziokulturelle Entwicklung des Landes stand im Kontrast zur Unterdrückung eines politischen Pluralismus im Iran, der zudem als amerikanischer Satellitenstaat wahrgenommen wurde. ${ }^{52}$ Die wachsende militärische Stärke des Iran wurde gar genutzt, um iranische Soldaten in arabischen Ländern im Auftrag und gemäß den vorgegebenen Interessen der Westmächte einzusetzen und damit sozusagen als Polizei der Region zu fungieren. ${ }^{53}$ Vorerst gescheitert schien damit nicht nur die Befreiung von imperialistischer Fremdbestimmung, nunmehr nicht von der britischen, sondern primär von der amerikanischen Regierung ausgehend, sondern auch von autoritärer und korrumpierter Staatsführung. Der Staatsstreich durch die USA 1953 steht so für einen dauerhaften Bruch mit den breiten gesellschaftlichen Kräften im Iran, die sich eine freiheitliche Ordnung bereits selbst erkämpft hatten, so dass bei jedem weiteren Engagement der US-Regierung bis heute der Vorwurf „imperialistischer Einflussnahme“ im Raum steht und politisch dienstbar gemacht werden kann.

Vgl. den „Ajax“-Operationsplan, Petherick (Fn. 37), S. 93 ff. und Kinzer (Fn. 23), S. 32 ff., 42, 237 ff. wo sehr detailliert die Einzelheiten der Kampagnen dargestellt werden.

Ebert/Fürtig/Müller (Fn. 31), S. 46; Prashad (Fn. 37), S. 77; Kinzer (Fn. 23), S. 270 f.

Ebert/Fürtig/Müller (Fn. 31), S. 83.

Perthes, Iran - Eine politische Herausforderung, 2009, S. 89; Nairn, Viva La Revolution! The Story of People Power in 30 Revolutions, 2012, S. 31.

Bayat-Philipp, Die Beziehungen zwischen den USA und Iran seit 1953, APuZ 2004, S. 29 (32).

Schweizer (Fn. 23), S. 267; Kinzer (Fn. 23), S. 272 f.

Bayat-Philipp (Fn. 50), S. 29 (33).

Bayat-Philipp (Fn. 50), S. 29 (33). 
Der aus strategischen Gründen erfolgte Sturz der demokratischen Regierung von Mossadegh 1953 stellt den Beginn einer Entwicklung dar, die letztlich zur Islamischen Revolution als endgültigem postkolonialen Befreiungsschlag führte. ${ }^{54}$

\section{Islamische Revolution als postkolonialer Befreiungsakt}

Wie auch in Südamerika und Afrika bedeutete das formale Ende von Kolonialismus und Imperialismus nicht das Ende von Abhängigkeit und Bevormundung. ${ }^{55}$ Die Rolle der Religion und der Einfluss der Religionsgelehrten auf die politische Gemeinschaft war - wie gesehen - im Iran nie zu unterschätzen, und so ist es letztlich nicht mehr verwunderlich, dass gerade sie das finale Aufbegehren gegen den immer despotischer agierenden Schah und die externe Einflussnahme steuerten. Wenngleich hier nicht der gesamte Vorgang der Islamischen Revolution analysiert werden kann, so ist doch die Absetzung des Schah und die Etablierung einer neuen Verfassung nach dem revolutionären Umsturz auch eine deutlich anti-imperialistische Bewegung, die die Einordnung in einen postkolonialen Kontext erlaubt. Die Revolution war zwar eigentlich in vorderster Linie gegen den Machthaber im Land gerichtet, doch da dessen Schicksal derart eng mit der amerikanischen Regierung verwoben war, ist eine auch hiergegen gerichtete Stoßrichtung der Revolution offensichtlich. Mithin ist Emmanuel Todd zuzustimmen, dass die Islamische Revolution von 1979 bereits in anderer Form das vorweg nimmt, was in den aktuellen revolutionären Umwälzungen im arabischen Raum passiert. ${ }^{56}$ Die Aufstände gegen die despotischen Verhältnisse in den arabischen Staaten Nordafrikas lassen sich so möglicherweise als verspäteten Widerstand gegen den Kolonialismus, der diese Zustände ermöglichte, lesen, aber vor allem als Widerstand gegen die innerstaatliche Tyrannei als Erbe des kolonialen Systems, das zudem als Teil eines dysfunktionalen globalen Wirtschaftssystems funktioniert, wodurch wirtschaftliche Rückständigkeit und Armut eher gefördert denn beseitigt wird. ${ }^{57}$ Ausgangspunkt für die revolutionären Bestrebungen mag auch ein demographisches Moment sein, junge Gesellschaften, die ihre Perspektive suchen, doch vor allem hängt eine politische Emanzipationsbewegung von einem hohen Bildungsniveau ab, das im Iran in den 70er Jahren ironischerweise gerade auch bedingt durch die Bildungspolitik des Schah - bereits erreicht war und folglich zum Aufbegehren gegen das repressive System führte. ${ }^{58}$ Die revolutionäre Bewegung, die sich unter der Führung der Religionsgelehrten, dabei natürlich vor allem Ayatollah Ruhollah Khomeini, vollzog, aber auch unter Einbeziehung der Linken und der nationalen Bewegung, die sich noch den Prinzipien von Mossadegh verpflichtet fühlte, sah sich bald einem, zuweilen brutalen, Exklusionsprozess ausgesetzt, der das von Khomeini ausgearbeitete Konzept eines Islamischen Staates, der Islamischen Republik Iran in den Vordergrund rückte. ${ }^{59}$ Dies vermag aber dem revolutionären Akt indes nicht pire, 2009, S. 9 (12).

Todd, Frei! Der arabische Frühling und was er für die Welt bedeutet, 2011, S. 18.

Hardy, The Muslim Revolt, 2010, S. 4 betont genau diese vielgestaltige Auseinandersetzung; vgl. auch Dabashi, Die Idee der Demokratie wird jetzt von anderen neu definiert, qantara v. 24.5.2011, www.qantara.de (1.3.2012).

Todd (Fn. 56), S. $23 \mathrm{f}$.

Vgl. grundlegend Khomeinis Schrift „Der islamische Staat , in deutscher Übersetzung erschienen 1983. 
seine Legitimation zu nehmen. Hier offenbart sich auch die Ebene, auf der Foucault, ebenso wie viele andere westliche Beobachter, 1979 argumentierte, der sich aus einer zeitgenössischen Betrachtung heraus dafür begeisterte, das ein Volk ohne weitreichende militärische und organisatorische Mittel gegen eine hochgerüstete Staatsmacht kämpfte, während die Religion eine wesentliche Grundlage bildet, deren strategische Relevanz Foucault erkannte, wenn er sie als „politische Spiritualität“ einordnete. ${ }^{60}$ Die von den Revolutionären „erträumte Geschichte“ hatte so gleichermaßen politischen wie religiösen Charakter, ${ }^{61}$ trägt also eine Glaubensmaximen verpflichtete politische Ideologie in sich. Nur weil aus einer Post-9/11-Sicht nahezu jeder Bezug eines Staates zum Islam im Kontext einer Bedrohung durch Islamismus und Terrorismus gesehen wird, ändert dies nichts an der Einordnung der Islamischen Revolution als postkolonialem Befreiungsakt. Auch der Vorwurf an Foucault, die islamische Revolution zu naiv betrachtet zu haben, scheint unverständlich, da er ebenfalls 1979 die der Revolution inhärente und sich später auch realisierende Ambivalenz benennt, indem er der islamischen Bewegung im Iran eine intensive und komplexe Realität attestiert, die „Bedeutendes“ und „Abscheuliches“ hervorgebracht habe. ${ }^{62}$ Mithin äußerte sich gerade im Kontext der islamischen Revolution die Hoffnung auf Revitalisierung der islamischen Zivilisation mit Blick auf zeitgenössische („moderne“) Gesellschaftsstrukturen und unter Einbeziehung der geistigen Errungenschaften von West und Ost, Okzident und Orient. ${ }^{63}$ Die dunklen Seiten von Revolutionen auf der ganzen Welt, so bedeutend die Revolutionen für den Moment und aus einer historischen Sicht sein mögen, sind bekannt und kamen auch hier zum Tragen. Die islamische Revolution und insbesondere Khomeini selbst kamen politisch auch in Verruf durch die Geiselnahme amerikanischer Botschaftsangehöriger. ${ }^{64}$ Angesichts der Vorkommnisse um den Putsch von 1953 ist die Mobilisierung iranischer Bürger für die Besetzung der amerikanischen Botschaft im Zuge der islamischen Revolution - zumal die amerikanische Regierung dem gestürzten Schah die Einreise gestattete - aus einer internen Sicht zumindest nachvollziehbar, da dort eine Keimzelle des Putsches von 1953 lag, was auch im kollektiven Gedächtnis der iranischen Bevölkerung präsent blieb. ${ }^{65}$ Das Verhältnis zur Regierung der USA war damit allerdings endgültig zerrüttet.

\section{Blutiges Nachspiel}

Aus iranischer Sicht hat sich das Szenario einer andauernden Bedrohung von außen sodann durch den unmittelbar auf die Islamische Revolution folgenden Angriff des Irak auf den Iran 1980 bestätigt, der ohne weitreichende militärische Unterstützung von Seiten der USA wohl kaum in dieser Form möglich gewesen wäre. ${ }^{66}$ Die überaus zurückhaltende Reaktion des UN-Sicherheitsrates zielte selbst nach einer Woche des laufenden Krieges noch nicht auf einen Waffenstillstand und vermochte den Aggressor als Bedrohung für den Internationalen Frieden und die Sicherheit nicht zu benennen. ${ }^{67}$ Die internationale Gemeinschaft als

Foucault, Nutzlos, sich zu erheben, in: Foucault, Analytik der Macht, 2005, S. 175 (176f.); Lemke, Nachwort. Geschichte und Erfahrung. Michel Foucault und die Spuren der Macht, in: Foucault, Analytik der Macht, 2005, S. 319 (343).

61 Foucault (Fn. 60), S. 175 (176).

62 Foucault (Fn. 60), S. 175 (177).

63 Kapferer (Fn. 16), S. 3; Foucault (Fn. 60), S. 175 (177).

64 Nairn (Fn. 49), S. 31.

65 Kinzer (Fn. 23), S. 281.

66 Kinzer (Fn. 23), S. 281.

67 Zarif, Tackling the Iran-U.S. Crisis: The need for a Paradigm Shift, in: Journal of International Affairs, 2007, S. 74. 
politisches Konstrukt war damit aus Sicht des Iran diskreditiert. Der achtjährige

Krieg forderte unzählige Opfer, wurde mit Giftgas- und Raketenangriffen auch in die Hauptstadt Teheran getragen und vermochte weite Teile der iranischen Bevölkerung durch die Fokussierung auf einen nationalen Patriotismus zu einen und so für eine Zeit lang den regimebezogenen Dissenz zu minimieren. ${ }^{68}$ Ein solches Szenario kann sich wiederholen, und ein Teil des kollektiven Gedächtnisses im Iran trägt noch den Verlust, den viele Familien in den Revolutions- und Kriegsjahren zu erleiden hatten, mit sich. So offenbart sich das abwehrende Element gesellschaftlicher Selbstbestimmung als das einigende Band gegen jedwede auswärtige Intervention. Auch wenn der Iran im 20. und 21. Jahrhundert keine kriegerische Auseinandersetzung gegen einen Nachbarstaat begonnen hat, bleibt die Sicherheitslage in Westasien fragil. Die Gewähr der Sicherheit des Landes und seiner territorialen Integrität bleibt von entscheidender Bedeutung und steht angesichts der veränderten sicherheitspolitischen Situation in Asien bzw. im Umfeld des Iran vor neuen Herausforderungen. Eine regionale Sicherheitsarchitektur hätte ein vielversprechender Ansatz sein können, der jedoch nicht weiter verfolgt wurde. ${ }^{69}$ Die außenpolitischen Erfahrungen seit 1953 lassen indes die skeptische Haltung der iranischen Regierung in Sicherheitsfragen deutlich werden. Doch der Abwehrreflex der Bevölkerung kann sich, das zeigt die revolutionäre iranische Tradition, ebenso abermals gegen innere Bevormundung und Unterdrückung richten. ${ }^{70}$

\section{Lesarten postkolonialer Selbstwerdung}

Der Iran stützt sich auf sein Selbstverständnis als Regionalmacht, die sich durch Eigenständigkeit und Streben nach Sicherheit definiert.

\section{Konstitutionalisierung von Autonomie}

Die Bedeutung historischer Bezüge offenbart sich im Bedürfnis, die eigene Geschichte selbst zu erzählen. Die Präambel der Verfassung des Iran, wohl eine der längsten Präambeln der Welt, ist hierfür symptomatisch, denn sie enthält über viele Seiten die Erzählung der Revolution, die ausdrücklich auch die Unterstützung der amerikanischen Regierung für das despotische Regime des Schah anprangert und sich dagegen verwahrt. Art. 3 Nr. 5 der Verfassung konkretisiert dies dahingehend, dass Aufgabe der Regierung des Iran „eine vollständige Beseitigung fremder Einflüsse“ sei. Konkretisiert wird dies durch Art. 43 Nr. 8 im Kapitel 4 der Verfassung zu Wirtschaft und Finanzen, der die Verhinderung von Fremdherrschaft über die Wirtschaft des Landes verlangt, und den im Kapitel 10 zur Außenpolitik verankerten Art. 153 der Verfassung, der ein Verbot von Abkommen, die die „Ausübung von Fremdherrschaft über die natürlichen Ressourcen die Wirtschaft, die Kultur, die Armee und andere Bereiche des Landes“ ermöglichen würden, konstituiert. Art. 146 verbietet zudem das Anlegen ausländischer Militärbasen auch zu friedlichen Zwecken. Diese Normen, ähnlich dem Art. 81 der iranischen Verfassung, der ausdrücklich die Konzessionsvergabe an Ausländer in bestimmten Bereichen verbietet, folgen schon in ihrem Vokabular einem anti-kolonialen bzw. anti-imperialistischen Impuls, wie er sich be- 
reits in der Rede von Mossadegh vor dem UN-Sicherheitsrat 1951 zeigte. Zugleich bescheinigt die Regierungszeit des ehemaligen iranischen Präsidenten Khatami, dass eine dialogfreundliche und reformorientierte Politik möglich ${ }^{71}$ und vor allem von weiten Teilen der iranischen Bevölkerung gewollt ist, wenngleich gerade in seine Regierungszeit die Einordnung des Iran in die „Achse des Bösen“ durch den amerikanischen Präsidenten erfolgte, was Khatami auch innenpolitisch mit seinem ohnehin fragilen Reformkurs entscheidend schwächte. ${ }^{72}$ Die durchaus starke gesellschaftliche Offenheit gegenüber Errungenschaften anderer Kulturen und Gesellschaften muss nicht weiter betont werden. Dies wird letztlich auch von der Verfassung des Landes insoweit unterstützt und eingefordert, als nach Art. 2 Nr. 2 die „Nutzung von Wissenschaft, Technik und Fortschritt menschlicher Erfahrungen und das Bemühen, sich weiter zu entwickeln, “ vom Staat, allerdings stets in Einklang mit einer politischen, wirtschaftlichen und sozio-kulturellen Unabhängigkeit, verlangt wird. Ergänzend garantiert Art. 3 Nr. 3 eine „unentgeltliche Bildung“ auf allen Gebieten, die der „Allgemeinheit zugänglich zu machen“ ist, und Art. 3 Nr. 4 verlangt vom Staat, den „Forschergeist“ auf allen Gebieten zu motivieren. Und selbst die jetzige Staatsführung äußerte zur Überraschung vieler zuweilen offen eine Perspektive von politischen Beziehungen gerade mit den USA. ${ }^{73}$

\section{Aspekte des islamisch-republikanischen Verfassungssystems}

Es etablierte sich mit der Islamischen Republik Iran im Nachgang der islamischen Revolution neben der Durchsetzung einer nationalen Eigenständigkeit immerhin auch ein Verfassungssystem, wonach die Bürger, im Gegensatz zu vielen ihrer Nachbarländer, den Präsidenten und das Parlament wählen können. ${ }^{74}$ Das Normengefüge der iranischen Verfassung enthält mit dem Parlament als einem politischen Entscheidungsträger, der Trennung zwischen Staatspräsident und den Rechtsgelehrten bzw. dem Wächter- und dem Expertenrat als einem gewaltenteilenden und -kontrollierenden System (vgl. Art. 56 ff., 122 und 134 Abs. 3 Verfassung Iran) durchaus bekannte Raster staatlicher Verfasstheit, wenngleich der ideologische Zuschnitt mit der klaren Ausrichtung sämtlichen staatlichen Handelns am islamischen Recht und der sich daraus ergebenden alternativen Institutionalisierung, die islamischen Rechtsgelehrten besondere Kompetenzen einräumt (vgl. z.B. Art. 5 Verfassung Iran), gänzlich differiert. Die Verfassung des Iran ist zugleich als Gesetz und Programm ${ }^{75} \mathrm{zu}$ verstehen, das eine islamische Ethik im Zusammenspiel mit der sozio-kulturellen Entwicklung des Landes, damit also einen sozio-politischen Kodex festschreibt, wie er letztlich auch schon in der Verfassung von 1905/07 angelegt war. Damit offenbart sich der Zusammenhang zwischen den drei revolutionären Aufständen gegen eine Unterdrückung durch absolute Monarchie und ausländische Interventionen. ${ }^{76}$ Der jetzigen Verfassung als normativer Ordnung wird dabei von unterschiedlicher Seite das Potential für die Gewähr eines funktionierenden islamisch-republikanischen

So initiierte Khatami bei der UN z.B. das Projekt „Dialogue among Civilizations“, vgl. Peterson, Let the Sword Encircle Me. Iran - A Journey behind the Headlines, 2010, S. 117; Annan, Brücken in die Zukunft. Eine Initiative, 2001, S. 9.

72 Perthes (Fn. 49), S. 26; Molavi (Fn. 28), S. 193.

73 Perthes, Iran als außenpolitischer Akteur, APuZ 2009, S. 3 (5).

74 Nairn (Fn. 49), S. 26, vgl. Art. 62 und Art. 114 Verfassung Iran.

$75 \mathrm{Zu}$ Lesarten von Verfassungstypen, die hier aber nur einen Ansatz vermitteln, vgl. Frankenberg, Autorität und Integration, 2003, S. $119 \mathrm{ff}$.

76 Mackey, The Iranians. Persia, Isiam and the Soul of a Nation, 1998, S. 272, 277. 
Systems zugestanden. ${ }^{77}$ Es ist zu hoffen, dass von dem bildungsstarken Potential der jungen iranischen Gesellschaft mehr und mithin entscheidende Impulse in den politischen Bereich hinein getragen werden, ohne die langjährige revolutionäre Tradition wachrufen zu müssen. Die Reklamation von Freiheit und Gerechtigkeit mit politischer Beteiligung aller am Gemeinwesen - und damit letztlich die essentiellen Forderungen bisheriger revolutionärer Bewegungen - findet im Grunde ebenfalls seine Stütze in der iranischen Verfassung, die nach Art. 2 Nr. 3 die Ablehnung jeder Unterdrückung gebietet und gemäß Art. 3 Nr. 8 die Beteiligung der gesamten Bevölkerung an der Gestaltung des Gemeinwesens einfordert. Entsprechende Bestrebungen sind allerdings nicht einfach mit den stereotypen Kategorien „,islamisch/religiös vs. säkular“ zu erklären, vielmehr findet diese Auseinandersetzung auch um die zeitgemäßen Lesarten des Islam statt. So sind es auch Religionsgelehrte, die die großen Teile des Volkes in ihrem Streben nach Gerechtigkeit, Transparenz der politischen Führung und politischer Mitbestimmung unterstützen, indem sie eine ideologische Basis für ihre Forderungen bieten, die nicht notwendig säkular ist. ${ }^{78}$ Die Anerkennung eines neuen, eines anderen Konstitutionalismus heißt auch für die internationale Gemeinschaft Entwicklungen zuzulassen, die jenseits des eigenen Systemdenkens liegen. ${ }^{79}$ 